\title{
Long-acting reversible methods of contraception: unified training
}

\author{
Amanda Britton, ${ }^{1}$ Anne Connolly ${ }^{2}$
}

\begin{abstract}
${ }^{1}$ GP Principal, Hackwood Partnership, Basingstoke, UK and Associate Specialist in Sexual and Reproductive Healthcare

${ }^{2}$ GPwSI in Gynaecology, The Ridge Medical Practice, Bradford, UK
\end{abstract}

\section{Correspondence to} Dr Amanda Britton, Hackwood Partnership, Essex House, Essex Road, Basingstoke, Hants RG21 8SU, UK ; amandabritton@nhs.net

Received 23 September 2013 Accepted 10 February 2014
To cite: Britton $A$, Connolly A. J Fam Plann Reprod Health Care 2014:40:80-81.

\section{OVERVIEW}

Improving access to long-acting reversible contraception (LARC) methods is an essential aspect in the work of reducing unwanted and unplanned pregnancies. This requires easy access to an appropriately trained workforce. Working together with the Royal College of General Practitioners (RCGP) and the Royal College of Nurses (RCN), the Faculty of Sexual \& Reproductive Healthcare (FSRH) has enabled both nurses and doctors to gain qualifications for the provision of LARC without necessarily holding Diplomate status within the Faculty.

\section{LARC PROVISION}

Holistic delivery of contraception includes being able to offer and deliver a full choice of contraceptives. The LARC methods include subdermal implants, injectable progestogens and intrauterine contraceptives [copper-containing intrauterine devices (IUDs) and the levonorgestrel-releasing intrauterine system (IUS)]. The particular advantages of these LARC methods include their reliability and effectiveness. In 2005, the National Institute for Health and Care Excellence (NICE) ${ }^{1}$ recognised LARCs as being the most cost-effective contraceptive methods, and for these reasons the promotion of such methods was encouraged. The importance of ensuring comprehensive and readily available training for health care professionals was highlighted. Having a substantial number of trained implant and IUD fitters enables good access to, and availability of, LARC methods for women and also ensures good quality patient care.

\section{ASSESSMENT OF COMPETENCY}

The competency of LARC fitters is acknowledged in different ways, by holding a Letter of Competence in subdermal implants (LoC SDI) or in intrauterine techniques (LoC IUT) as awarded by the FSRH, or with a qualification awarded by the RCN or from a locally devised competency assessment. Some commissioners also recognise individual competency under a 'grandfather clause', namely that of an individual who has been practising the technique for a number of years.

For women accessing these contraceptive methods it is important that a recognised level of expertise of the fitter is quality assured and recertified when applicable. That there is a risk associated with these procedures was highlighted by the media scare in 2011 when cases were identified following problems caused by poor SDI insertion training and failure to fit these devices correctly. Unfortunately failure of correct insertion caused distress to women and also had a detrimental effect on the LARC uptake campaign at the time.

\section{INDEPENDENT REVIEW OF THE} DFSRH

In 2011 the FSRH commissioned an independent review of the Diploma of the Faculty of Sexual and Reproductive Healthcare (DFSRH) that had been launched in 2010, and also of the Letters of Competence. Professor Peile made 27 major recommendations in his report, ${ }^{2}$ the majority of which have been addressed under three areas: a review of access to training for nurses and doctors, streamlining the training process, and enabling individuals to achieve LoCs without necessarily holding the DFSRH or paying an annual subscription to the FSRH. The latter stipulation had been a concern for those individuals who were members of other faculties and colleges. An invited multidisciplinary group known as the 'Quality Training Project' was established and worked with other institutions, in particular the RCGP and the RCN, to explore these proposals.

\section{LOC ENTRY REQUIREMENTS}

The Faculty has now created standalone LoCs for doctors and nurses to access 
and self-certify. In recognition of the importance of the contraceptive choice consultation prior to using a LARC method and of the need to be able to manage related complications, an online knowledge assessment - the 'eKA' - is one of the entry points to the practical assessment. This can be accessed from any computer with Internet access, and assesses the knowledge required to deliver non-specialist sexual and reproductive health care including LARC methods in primary and community care. The questions are based on typical clinical scenarios and are designed to evaluate the extent of the candidate's knowledge and clinical judgement in the areas in which a clinician providing LARC methods would be expected to demonstrate a high level of competence. Other entry requirements that are either self-certified or signed off by a trainer include consultation skills and, in the case of the LoC IUT, the ability to perform a bimanual pelvic examination. The practical elements of the LoCs remain unchanged and include training using models and, in the case of subdermal implants, two consecutive insertions/removals being deemed competent, as a requirement before certification. In order to gain the LoC IUT, individuals new to IUD/IUS insertion need to insert a minimum of seven devices (including copper-containing IUDs and the IUS) under supervision, while those with previous insertion experience may self-certify five of their minimum of seven insertions.

Assessing practical skills and competencies is time consuming and needs prior organisation. With clinical time pressures and changes in commissioning of services it is important to ensure that opportunities to access such training are made as easy as possible. FSRH registered trainers (FRTs) are recognised by the Faculty for the training and assessment of LARC providers and include both doctors and nurses. General practitioner (GP) trainers who already hold the appropriate LoC can be recognised as FRTs, and initiatives to recognise this GP cohort will be important in helping to address future training requirements. Details of local trainers can be obtained from local General Training Programme Directors whose details can be found on the Faculty website (http://www. fsrh.org).

LoC SDI and LoC IUT are recognised by most commissioners of sexual health services. They are nationally transferable and must be recertified every 5 years. Trained clinicians who hold LoCs but do not hold the FSRH Diploma may now choose whether to have the additional advantages of becoming an associate member of the FSRH by paying an annual subscription. This would support their updating and specialist education and includes receipt of the quarterly journal and other benefits. They could also choose to build on their skills and to work towards gaining a Diploma of the FRSH, which is now open to both doctors and nurses.

\section{THE FUTURE}

These are exciting times for the FSRH as this initiative is important in unifying and standardising the training of doctors and nurses. Nurses who have already been accredited by the RCN to fit LARCs have been offered a LoC as equivalence and they can transfer the ability to recertify via the FSRH. For doctors and nurses who are in sole possession of a local LARC fitting competency certificate there is the opportunity to gain a LoC by fulfilling the requirements outlined above. For experienced providers the required review of their technique by an experienced trainer will hold the positive benefits of peer review. This would count as educational hours towards the requirements of annual appraisal and revalidation.

Details of this important initiative to streamline the training of LARC fitters are documented on the FSRH website (http://www.fsrh.org). These qualifications for providing LARC have been accredited by both the RCGP and the RCN.

\section{Competing interests None.}

Provenance and peer review Not commissioned; externally peer reviewed.

\section{REFERENCES}

1 National Institute for Health and Care Excellence. Long-Acting Reversible Contraception (NICE Clinical Guideline 30). http:// guidance.nice.org.uk/CG30 [accessed 2 October 2013].

2 The Independent Evaluation of the Diploma of the Faculty of Sexual and Reproductive Health (DFSRH) and the 'Letter of Competence' training programmes - Recommendations. http:// www.fsrh.org/pdfs/DFSRH_EvaluationRecommendations.pdf [accessed 2 October 2013]. 